\title{
Éditorial
}

\section{Prendre des risques: un besoin oublié!}

Prendre des risques c'est affirmer sa liberté. Et pourtant nous, qui considérons la liberté comme la pierre angulaire de notre société, avons terriblement peur du risque. C'est l'un des paradoxes qui nous est propre: affirmer la liberté des individus, et, en même temps, détester ce qui n'est pas planifié ou prévu. Or, " c'est seulement par le risque de sa vie que l'on conserve la liberté » (Hegel, 1807). Selon ce philosophe, c'est par le risque de sa vie que l'homme émerge de son animalité pour accéder à l'humanité. Bien sûr il ne s'agit pas là d'une invitation à aller guerroyer, mais bien d'une invitation à prendre sa vie en mains et à ne pas avoir peur de vivre.

Cette peur de vivre, trop de personnes âgées la connaissent et nous, professionnels de la santé et intervenants de toutes sortes, y avons une part de responsabilité. Il est naturel pour nous de vouloir protéger ceux qui nous semblent plus fragiles, plus à risques d'être lésés. Il est de l'essence même du travail professionnel de minimiser les risques. Mais lorsque l'atténuation des risques prend toute la place, cela peut avoir pour effet de brimer la liberté des personnes. C'est ce que sous-tend l'article de Michel Silberfeld. Cette phrase tirée de la conclusion illustre ce propos: « Starting from assuredly good intentions, risk becomes part of unconscious rhetoric for controlling elderly people. "

L'évaluation des risques est certes un exercice utile au niveau collectif; il faut toutefois faire preuve de beaucoup de prudence lorsqu'appliquée au niveau individuel. Comme le souligne Silberfeld, il faut prendre garde d'appliquer aux individus des conclusions tirées de modèles actuariels. Ces modèles sont utiles pour établir des politiques générales, mais ne nous permettent pas de poser des jugements objectifs au niveau d'un individu. La science clinique ne nous est, d'ailleurs, guère plus utile. Distinguer ce qui est désirable de ce qui ne l'est pas ne relève pas d'elle. Elle ne peut que contribuer à voir les issues possibles et souvent de façon fort limitée puisque l'art du pronostic n'est pas des plus faciles. De plus, l'évaluation d'un risque doit tenir compte non seulement du risque lui-même mais de la perception qu'en a la personne. Cette perception est aussi importante que le risque lui-même puisque c'est elle qui déterminera le choix final. Elle le détermine d'autant plus qu'elle est elle-même influencée par les pressions de l'entourage et les moeurs de la collectivité. L'évaluation des risques est donc loin d'être un exercice objectif.

En plus d'être conscients de notre peu de capacités à établir la probabilité qu'un risque particulier se matérialise chez un individu, nous devons être conscients de notre peu de capacité à mettre de côté une échelle de valeurs 
professionnelles où le bien-être de la personne tient plus à coeur que le respect de ses choix. Cette échelle de valeur a de profonde racine. Malgré tous les discours sur le respect de l'autonomie individuelle, cette dernière n'a pas beaucoup de place au sein des relations professionnelles que nous entretenons avec les personnes âgées. La recherche d'un équilibre entre le principe d'autonomie (autonomy) et le principe de bienfaisance (beneficence) est l'objet même de tout débat éthique. Il est cetain qu'il y a eu à ce chapitre évolution des mentalités; il n'en reste pas moins vrai qu'en ce qui concerne les personnes âgées, la balance penche beaucoup plus souvent au détriment du libre choix des individus. Nos interventions cliniques devraient être bien davantage inspirées par une présomption en faveur de l'autonomie des personnes que par une présomption en faveur de ce que nous croyons bien pour elles.

L'implication des familles est un aspect trop souvent négligé. Lorsque l'on intervient auprès de personnes âgées qui ont perdu une partie de leur autonomie fonctionnelle, il est primordial de " mettre la famille dans le coup ". C'est souvent par ce moyen que nous pouvons le mieux comprendre les besoins des personnes âgées. Cela ne veut pas dire qu'il revient à la famille de décider ce qu'il convient de faire à la place de la personne âgée. Cela veut plutôt dire qu'il faut considérer la famille comme étant notre client tout autant que la personne âgée elle-même. Elle doit bénéficier de notre attention et de notre aide. Cette compréhension et cette aide, elle en a d'avantage besoin lorsque la personne âgée a quelques difficultés cognitives. Si ces difficultés sont telles qu'elles peuvent faire naître un doute sur la compétence mentale de la personne âgée, il est alors nécessaire d'évaluer cette compétence.

Comme le souligne Silberfeld, l'évaluation de la compétence mentale d'une personne n'est pas chose facile. Il faut d'abord avoir à l'esprit qu'une personne est présumée compétente jusqu'à ce que l'on démontre clairement qu'elle ne l'est plus. Cela est vrai autant d'un point de vue éthique que d'un point de vue juridique, autant en droit civil qu'en common law. Or, il n'y a pas de test standardisé qui permette d'établir clairement qu'une personne n'est plus compétente. La compétence n'est pas une question de tout ou rien; elle doit être évaluée en fonction de la nature de la décision à prendre. La compétence requise pour prendre une décision en matière de résidence n'est certainement pas la même que celle qui serait requise, par exemple, en matière de gestion financière ou encore, en matière de procès. Bien que les instruments d'évaluation lui fassent cruellement défaut, il revient malgré tout au clinicien de déterminer la compétence. Cette évaluation est, à notre avis, d'une importance plus grande que l'évaluation des risques. D'ailleurs, la recherche clinique devrait accentuer ses efforts pour développer des instruments d'évaluation plus précis. C'est dans cette direction qu'il faut prioriser la recherche plutôt que dans le développement d'instruments d'évaluation des risques, tout en étant conscients que " the search for a single test of competency is a search for a Holy Grail " (Roth, 1977).

Il n'est peut-être pas inutile de signaler au passage que lorsque la per- 
sonne âgée est incompétente, d'autres doivent décider pour elle. Dans ces situations, la famille prend encore plus de place. De fait, il lui revient de prendre les décisions au nom de la personne incompétente. Au Québec, les dernières modifications apportées au Code Civil confient clairement, lorsqu'il n'y a pas déja de représentant légal, les décisions de soins au conjoint ou aux proches parents. On donne ainsi une assise juridique claire au rôle que doit jouer la famille dans les décisions importantes. La famille est en général bien placée pour prendre de bonne décision. Toutefois, cela ne veut pas dire qu'alors l'évaluation des risques n'a plus sa place; cela veut plutôt dire que l'évaluation des risques a alors une autre préoccupation. En effet, autant il est légitime que la famile joue un rôle dans les décisions importantes, autant il est clair qu'elle ne peut faire courir de risques déraisonnables à la personne qui n'est pas en mesure de les apprécier. Lorsqu'une famille met en péril la sécurité d'une personne âgée, les professionnels ont le devoir de protéger la personne menacée. Inversement, lorsque la famille surestime les risques réels et réclame des professionels des mesures de sécurité exagérée, ces derniers doivent prendre le temps d'éclairer la famille. Les professionnels ont aussi le devoir de ne pas se rendre complice de mesures de sécurité susceptibles de brimer la personne âgée.

Les professionnels doivent donc trouver l'équilibre entre trois valeurs fondamentales; le respect de l'autonomie, la recherche de la bienfaisance et la protection des personnes vulnérables. Les deux dernières ont de solides assises culturelles. Celles de la première sont encore jeunes et fragiles. Quoiqu'on en dise, le respect de la liberté de choix du client n'est pas très ancré dans les moeurs des intervenants du monde des services de santé et des services sociaux. Le discours y est; les actes y sont encore peu présents. Peu d'entre nous sont conscients du fait que, pour être libre, l'être humain a besoin de risques autant que de sécurité. Supprimer tous les risques, c'est supprimer la vie elle-même. " Si l'on veut lutter contre la peur de vivre, il faudrait que les mentalités évoluent de telle façon que le besoin de risques puisse être pris en considération autant que le besoin de sécurité " (Dufresne, 1990). C'est là une proposition qui questionne profondément nos manières d'agir. Dans cette perspective, supprimer tous les risques c'est non seulement brimer la liberté, mais c'est aussi ne pas répondre adéquatement aux besoins des personnes. Une telle affirmation n'est certes pas une invitation à la négligence, mais très certainement, une invitation à parler plus ouvertement avec les personnes âgées de cet équilibre nécessaire entre risque et sécurité.

Le besoin de sécurité, c'est d'abord et avant tout une question de valorisation et de respect. Ce besoin sera satisfait si l'on parvient à assurer aux personnes âgées une place au sein du tissu social. C'est parce que la société met les aînés à l'écart qu'elle se sent la responsabilité de les préserver de tout risque, même au prix de leur liberté. Mais en réalité, ce n'est là qu'une façon de compenser notre profond sentiment de culpabilité. Rendons aux aînés leur véritable place, leur utilité sociale et nous aurons fait beaucoup plus pour leur sécurité que toutes les mesures possibles d'atténuation des 
risques. Car, pour finir par où nous avons commencé, il n'y a pas de véritable liberté sans risques.

\section{Références}

Dufresne, J. (1990). Gérontologie, interdisciplinarité: des mots qui peuvent devenir des maux. Actes $d u I^{\mathbb{Q}}$ Congrès international francophone de gérontologie. Edisem/Maloine.

Hegel, Georg Friedrich Wilhelm. (1807). Phénoménologie de l'Esprit. Édition Ambier.

Roth, L.H. (1977). Test of Competency to consent to Treatment. American Journal of Psychiatry, 134(3), 279.

Gilles Voyer, Hôpital d'Youville de Sherbrooke 